\title{
Das Arbeitsrecht der Zukunft im globalen Wettbewerb
}

Die Globalisierung hat die Politik der Unternehmen verändert. Zugleich haben sich auch die Bedürfnisse und das Verhalten der Menschen gewandelt - als Folge der Individualisierung und brüchig werdender sozialer Milieus. Die industriellen Beziehungen in Deutschland sind nicht mehr auf Konsens orientiert, die Verbände schwächeln. Das führt zu Funktionsstörungen des komplizierten Regelungssystems Arbeitsrecht. Seine herkömmlichen Schutzmechanismen können die neuen Formen der Beschäftigung und wirtschaftlichen Zusammenarbeit immer weniger erfassen, sie sehen sich weitgehenden Angriffen ausgesetzt. Jedoch bedarf es weiterhin des sozialen Ausgleichs und damit eines zukunftsfähigen Arbeitsrechts.

\section{Einleitung}

Juristen taugen nicht als Zukunftsforscher. Ihr methodisches Instrumentarium ist normgebunden. Es setzt voraus, dass konzeptionelle Vorgaben bereits vorhanden sind. Rechtspolitische oder privatautonome Entscheidungen bilden den klassischen Gegenstand juristischen Nachdenkens. Das bedeutet aber nicht, dass für Juristen nur Vergangenes Bedeutung hätte. Recht ist immer auch normativ strukturierte Planung. Und rechtliche Probleme bzw. Konflikte beruhen regelmäßig auf Planungsfehlern oder auf rechtlicher Fehlsteuerung. Die Lösung solcher Konflikte erfordert Kenntnis und Bewertung von Entwicklungen sowie Prognosen. Dabei geht es nicht allein um Planungssicherheit. Der demokratische Rechtsstaat verlangt darüber hinaus, dass seine Ordnung mit den Gerechtigkeitsvorstellungen der Gesellschaft vereinbar ist und in einem legitimierenden Verfahren gewonnen wird. Kennzeichen freiheitlicher Zivilgesellschaften sind demokratisch legitimierte und an Grundrechte gebundene Rahmenordnungen, die privatautonomen Gestaltungsformen möglichst breiten Raum lassen.

Das deutsche Arbeitsrecht zeigt, wie so etwas funktionieren kann. Differenziert auf drei Regelungsebenen - Arbeitsvertrag, Kollektivvertrag, Gesetz - ist es in den letzten hundert Jahren schrittweise in der Praxis entwickelt worden, wobei Gesetzgeber und Rechtsprechung immer erst nachträglich strukturierend, sanktionierend, oft auch korrigierend eingriffen. Diese naturwüchsige Praxisnähe war die große Stärke des Arbeitsrechts. Aber jetzt wird sie zum Problem.

Das Arbeitsleben befindet sich in einem rasanten und tief gehenden Strukturwandel. Die veränderten Rahmenbedingungen einer Wirtschaft im globalen Wettbewerb verlangen Anpassungen und Umstellungen. Dabei zeigen sich Funktionsstörungen des komplizierten Regelungssystems. Neue Formen der Beschäftigung und wirtschaftlichen Zusammenarbeit sind mit den Schutzmechanismen des Arbeitsrechts nicht mehr zu erfassen. Die alten Schutzund Regelungsprobleme des sozialen Ausgleichs bestehen aber nach wie vor, haben sich eher verschärft. Gibt es einen Wertewandel im Arbeitsrecht oder bedarf es gar eines solchen Wertewandels?

\section{Globalisierung und Wertewandel}

Das Ursachenbündel, das den tief gehenden Strukturwandel bewirkt hat und uns zu radikalem Umdenken zwingt, wird unter dem verschwommenen und phrasenhaften Begriff "Globalisierung" zusammengefasst. Das ist so bekannt, dass hier nur skizzenhaft auszuführen ist, worauf es für die folgenden Überlegungen besonders ankommt: Neue Technologien haben mit rasanter Geschwindigkeit die Möglichkeiten und Bedingungen von Güterproduktion, Dienstleistung und Zahlungsverkehr, ja die gesamten Wettbewerbsbedingungen erweitert und verändert. Menschliche Arbeitskraft ist kostengünstig ersetzbar, Erfahrungswissen und neue Erkenntnisse sind speicherbar und leicht verfügbar,
Kommunikation ist jederzeit und ohne räumliche Beschränkung möglich und führt zu einer globalen Vernetzung von Kontakten, Informationen und Ideen. Standortbindungen sind dadurch stark gelockert. Fast alles ist „weltmarktfähig“ geworden.

Schon diese rein wirtschaftliche Betrachtung zeigt, dass das Recht vor völlig neue Ordnungsaufgaben gestellt wird. Sie bedürfen zum großen Teil supranationaler Lösungen. Aber auch im nationalen Rahmen schafft das veränderte Konsum- und Kommunikationsverhalten der Bevölkerung eine Fülle neuer Regelungsfragen. Noch viel einschneidender sind die sozialen Probleme in der Folge einer grundlegend veränderten Arbeitswelt. Die Rechtsordnung kann und darf ihnen nicht ausweichen.

Die Suche nach angemessenen Lösungen hat zu beachten, dass das Recht nicht nur Ordnungsaufgaben wahrzunehmen hat, sondern Gerechtigkeitsvorstellungen und Erwartungen der Gesellschaft befriedigen muss. Auch diese sind aber in den globalen Wandlungsprozess hineingezogen. Die neuen Herausforderungen und Chancen beeinflussen das gesellschaftliche Leben und prägen auch Lebensentwürfe und Wertvorstellungen.

Wie stabil solche zeitgebundenen Erscheinungen sein werden, lässt sich kaum voraussagen. Nachhaltig werden aber mit Sicherheit Einflüsse wirken, die von unseren Nachbarländern ausgehen. Nicht nur die Marktteilnehmer stehen im Wettbewerb, sondern auch die Staaten mit ihren

Thomas Dieterich, Prof. Dr., Präsident des Bundesarbeitsgerichts a. $D$. 
spezifischen Rechtssystemen und Wertordnungen. Historisch gewachsene Überzeugungen und traditionelle Regelungsmechanismen geraten dadurch unter Begründungsdruck. So erscheint der „rheinische Kapitalismus" mit seinen typischen „checks and balances", trotz seiner großen Erfolge in der Vergangenheit, im Vergleich mit anderen Rechtssystemen nicht mehr über jeden Zweifel erhaben. Wie steht es mit seiner Effizienz?

\section{Entwicklungen und Funktionsstörungen der deutschen Arbeits- und Sozialordnung}

\subsection{INDIVIDUALARBEITSRECHT}

In Deutschland sind die gesellschaftlichen Wertvorstellungen in Bezug auf Solidarität und faire Verteilung der Lebensrisiken und -chancen sehr ausgeprägt. Das Arbeitsrecht ist normatives Substrat der entsprechenden Erwartungen und Schutzbedürfnisse. Für das Teilgebiet der Rechtsbeziehungen zwischen Unternehmen und ihren abhängig Beschäftigten kennzeichnet es den gesellschaftlich akzeptierten Interessenausgleich. Das nach und nach entstandene Gesamtkonzept stößt aber zunehmend auf Widerstände. Mit den Rahmenbedingungen verschieben sich auch die Machtverhältnisse.

\section{GEWACHSENE STRUKTUREN}

Der zentrale Ort des Arbeitslebens (für Arbeitgeber ebenso wie für Arbeitnehmer) war der Betrieb und ist es weitgehend auch heute noch. Seit Taylor und Ford war das eine straff organisierte und hierarchisch gegliederte Einheit. Der Arbeitgeber hat als deren Organisator und Leiter dafür zu sorgen, dass mit Hilfe von Menschen, Maschinen und Material ein marktfähiges Ergebnis erzielt werden kann. Holzschnittartig vereinfacht könnte man sagen, dass die Arbeitnehmer in diesem Modell nur als „Betriebsmittel" erscheinen. Sie schulden keinen Erfolg, sondern Fleiß und Gehorsam. Sie müssen „funktionieren“. Ihre kollektive Interessenwahrung hat das zwar im Laufe der Arbeiterbewegung abgemildert, und die gesetzliche Mitbestimmung hat die hierarchische Leitungsstruktur einem eman- zipatorischen Menschenbild näher gebracht, aber das hierarchische Organisationsmodell blieb prototypisch und für den Betriebsbegriff des Arbeitsrechts prägend.

Parallel und passend dazu hat sich das „Normalarbeitsverhältnis" als rechtliches Leitbild entwickelt. Es ist durch Weisungsgebundenheit gekennzeichnet, auf Dauer angelegt und vermittelt als Vollzeitbeschäftigung durch tariflich geregelte Löhne und Gehälter den Lebensstandard des (in der Regel männlichen) Arbeitnehmers und seiner Familie. In einer lückenlosen Berufsbiografie führt es zu einer solidarisch finanzierten Alterssicherung. Das Ideal dieser Rechtsbeziehung ist Verlässlichkeit und Dauer. Daran waren auch die Unternehmen ursprünglich stark interessiert. Sie benötigten eine zuverlässig eingespielte $\mathrm{Be}$ legschaft. Starke Betriebsbindung galt als Wettbewerbsvorteil. Mit erheblichem Kostenaufwand wurden dafür freiwillige Leistungen und betriebliche Einrichtungen zur Verfügung gestellt.

\section{WANDEL DER FUNKTIONS- GRUNDLAGEN}

Der globalisierte Wettbewerb hat diese Sicht der Unternehmen drastisch verändert. Die neuen Möglichkeiten der Rationalisierung von Arbeitsprozessen entfalteten unter dem Druck der Konkurrenz und der Finanzmärkte schon bald drängende Sachzwänge mit der Folge eines tief greifenden Strukturwandels. Der Arbeitskräftebedarf hat sich verringert, sektoral verlagert und bezüglich seines Anforderungsprofils stark verschoben. Dass der soziale Rechtsstaat dies bisher ohne gesellschaftliche Eruptionen verkraften konnte, ist fast ein Wunder. Allerdings hat er seine Steuerungseuphorie dabei mehr und mehr verloren. Seine Reaktionen erinnern eher an „kontrolliertes Schleudern“. Inzwischen ist die Rallye keineswegs beendet, ihr Tempo unvermindert und die Streckenführung hält sich nicht mehr an die vertraute Topografie des Arbeitsrechts. Beispiele hierfür sind:

(1) Die Betriebsorganisation: Hier sind die Veränderungen am auffälligsten. Der alte „fordistische" Betrieb hat sich als zu voluminös und schwerfällig erwiesen; seine zentralistische Leitungsstruktur erscheint unzeitgemäß. Stattdessen versuchen die Unternehmen mithilfe kleiner dezentraler Einheiten, die oft sogar rechtlich verselbst- ständigt werden, Entscheidungs- und Arbeitsprozesse stärker zu verteilen und deren Zusammenspiel so zu organisieren, dass eine „denkende Fabrik“ entsteht. Das funktioniert nur bei flachen Hierarchien und vernetzter Kommunikation. Hingegen ist der lokale Standort der Leitungsebene und der einzelnen Mitarbeiter oft ziemlich gleichgültig, die örtliche Nähe und räumliche Zusammenfassung wird zunehmend entbehrlich. Im „virtuellen Betrieb“ treffen sich die Mitarbeiter nur noch gelegentlich.

Aber für das deutsche Arbeitsrecht bietet der Betrieb in seiner traditionellen Form einen Referenzpunkt, der bei den unterschiedlichsten Aufgaben als Wertungsrahmen gilt. An ihm orientieren sich z. B. die Rechtsgrundlagen der betrieblichen Übung und der Gleichbehandlungsgrundsatz; der Betrieb bietet den Rahmen für die soziale Auswahl bei betriebsbedingten Kündigungen; er gilt als Bezugspunkt für den Geltungsbereich von Tarifverträgen und die Tarifzuständigkeit von Verbänden. Vor allem ist das Betriebsverfassungsrecht ihm ganz speziell gewidmet. Der Betrieb kennzeichnet die Legitimationsgrundlage des Betriebsrats und den Gegenstand seiner Mitwirkungsrechte. All dies verlangt nach klaren Grenzen und Entscheidungsebenen, die bei den heutigen Organisationsstrukturen verschwimmen. Rechtsunsicherheit und frustrierende Konflikte sind die Folge.

(2) Das Normalarbeitsverhältnis: Weniger sinnfällig, aber in den Konsequenzen viel einschneidender ist ein anderes Phänomen: Die Erosion des Normalarbeitsverhältnisses. Es handelt sich um einen schleichenden Prozess, der vor allem jüngere Beschäftigte betrifft und sich auch statistisch belegen lässt. Immer mehr Arbeitnehmer werden nur noch zeitlich befristet oder mit verringerter Arbeitszeit eingestellt oder „ausgeliehen“. Die volle Wucht der Entwicklung wird allerdings erst deutlich, wenn man nicht nur die Situation abhängig Beschäftigter vergleicht, sondern auch die neuen Formen von Selbstständigkeit einbezieht. Offensichtlich vermeiden die Unternehmen den Abschluss von Arbeitsverträgen soweit als irgend möglich und versuchen, die benötigte Arbeitsleistung in der Form von Dienst- und Werkverträgen zu gewinnen.

Die Erosion des Normalarbeitsverhältnisses und die Zunahme atypischer und pre- 
kärer Beschäftigung haben für das Arbeitsrecht zur Folge, dass seine Wirkung weitgehend leerzulaufen droht. Seine Grundvoraussetzung besteht ja darin, dass ein grundsätzlich auf Dauer angelegtes Arbeitsverhältnis überhaupt zustande kommt. Seine Regelungen (die gesetzlichen wie auch die kollektivvertraglichen) orientieren sich an den Schutzbedürfnissen des Normalarbeitsverhältnisses, verfehlen also in prekären und anders strukturierten Rechtsbeziehungen ihren Zweck ganz oder teilweise.

\subsection{KOLLEKTIVES ARBEITSRECHT}

Wie sehr die Globalisierung und die mit ihr verbundenen Entwicklungen die Praxis der industriellen Beziehungen antreiben und sie zwingen, den Status quo zu verändern, zeigt sich besonders deutlich, wenn man die ursprüngliche Konzeption des Gesetzgebers für das kollektive Arbeitsrecht mit der weiteren Entwicklung vergleicht.

\section{GESETZLICHE GRUNDLAGEN DES KOLLEKTIVEN ARBEITSRECHTS}

Das kollektive Arbeitsrecht sieht als gesetzgeberisches Konzept zwei scharf abgegrenzte Systeme der kollektiven Interessenwahrnehmung und Regelung vor, die sich in ihrem Einigungsverfahren, ihrer Legitimationsgrundlage und in ihrer Wirkungsweise grundsätzlich unterscheiden.

Die betriebliche Mitbestimmung ist ein Produkt staatlicher Rechtsgestaltung, das demokratische Prinzipien in das Privatrecht einführt. Der Betriebsrat vertritt als gewählter Repräsentant die ganze Belegschaft mit demokratischer Legitimation in den Grenzen gesetzlich geregelter Kompetenzen und Verhaltenspflichten. Dieser gesetzliche Rahmen ist nicht verhandelbar. Der angestrebte Interessenausgleich darf auch nicht mit Kampfmitteln erzwungen werden, sondern nur in vertrauensvollem Zusammenwirken zustande kommen. Dafür stellt der Gesetzgeber ein Einigungsstellenverfahren zur Verfügung. Insgesamt ein stark reguliertes, wirtschaftsfriedliches Modell mit bürokratischen Strukturen.

Auf der anderen Seite steht die Tarifautonomie, die aus dem Grundrecht der Koalitionsfreiheit (Art. 9 III GG) abgeleitet wird und durch Staatsferne gekennzeichnet ist. Gesetzgebung und Rechtsprechung sind zwar von Verfassungs wegen ver- pflichtet, ein funktionstaugliches Tarifvertragsrecht bereitzustellen und zu sichern, aber dessen Realisierung ist allein Sache der Tarifvertragsparteien. Ihnen bleibt es überlassen, wie sie durch geeignete organisations- und tarifpolitische Konzepte einen Mitgliederbestand erwerben und erhalten, der ausreicht, um ihre Interessen durchzusetzen - notfalls mit Druck und Gegendruck im Arbeitskampf. Eine Zwangsschlichtung findet nicht statt. Verhandlungsergebnisse sind auch im Prinzip nur für die beiderseitige Mitgliedschaft verbindlich (mit kleinen Ausnahmen und Auslauffristen).

Dieser zugespitzte Vergleich der beiden Systeme macht deutlich, dass ihre Vereinbarkeit keineswegs ganz unproblematisch ist. Die grundsätzliche gesetzliche Rangordnung ist klar: Der Schutz der Tarifautonomie steht ganz im Vordergrund; tariflichen Regelungen wird grundsätzlich der Vorrang eingeräumt. Das spezielle Gestaltungsmittel der Betriebsvereinbarung ist generell gesperrt, soweit Arbeitsbedingungen in Tarifverträgen geregelt sind oder üblicherweise geregelt werden. Das gilt sogar in Betrieben, die gar nicht tarifgebunden sind. Freiwillige Regelungsabsprachen zwischen Arbeitgeber und Betriebsrat bleiben allerdings unbenommen.

Eine Kooperation der beiden Systeme ist dennoch grundsätzlich erwünscht. Die Betriebspartner werden deshalb gesetzlich verpflichtet, mit den Tarifvertragsparteien zusammenzuwirken. Tarifverträge können ergänzende Betriebsvereinbarungen zulassen, sie können auch Abweichungen zuungunsten der Arbeitnehmer gestatten.

\section{PRAXIS DER KOOPERATION}

Die Erfahrung hat gezeigt, dass eine scharfe Trennung der beiden kollektiven Regelungssysteme sachgerechte Lösungen außerordentlich erschweren würde. Es besteht ein wachsendes Bedürfnis, ihre jeweiligen Stärken zu kombinieren und dadurch ihre Schwächen zu kompensieren. So erklärt es sich, dass das Bild der aktuellen Tarifpolitik geprägt ist von Kooperationsmodellen, die die betriebliche Mitbestimmung einbeziehen.

Am Beginn dieser Entwicklung stand das Bedürfnis der Tarifpraktiker, im Interesse größerer Beweglichkeit und Differenziertheit in Tarifverträgen lediglich Rahmen, Alternativen oder Ziele vorzugeben, hingegen den Betriebspartnern zu überlas- sen, wie sich diese Vorgaben mit den jeweils vorhandenen Gegebenheiten umsetzen lassen. Richtungweisend war hier der LeberKompromiss im Konflikt um die Arbeitszeitverkürzung. Das BAG hat die darin gefundene Kombination von betrieblichen und tarifvertraglichen Gestaltungsmitteln gebilligt (BAG 1987). Damit waren die beiden Systeme des kollektiven Interessenausgleichs rechtssystematisch verbunden, ihre Kooperation erscheint also trotz des Vorrangs der Tarifautonomie rechtlich gesichert.

\section{KREATIVE TARIFPOLITIK}

Für die weitere Entwicklung wichtig und folgenreich waren dann aber tarifpolitische Tendenzen, die das Tarifvertragsrecht inzwischen vollkommen verändert haben. Auch hier wird diese Entwicklung von den grundlegend andersartigen Bedingungen unternehmerischer Tätigkeit im globalen Wettbewerb angetrieben. Die regionalen und fachlichen Grenzen der Tarifgebiete verlieren ihre Wettbewerbsrelevanz, große Unterschiede in der Marktsituation der einzelnen Unternehmen sperren sich gegen einheitliche Regelungen der Sozialstandards und der Kostenbelastung, schnell wechselnde Rahmenbedingungen erzwingen kurzfristige Reaktionen. All das führt zu einem starken Bedürfnis nach Dezentralisierung und Flexibilität, dem sich die Tarifpolitik nicht entziehen kann. So sind in den letzten zehn, 15 Jahren vielfältige Formen der Kooperation und Kombination zwischen tariflichen und betrieblichen Regulierungssystemen zustande gekommen. Heute ist die Tariflandschaft bunt, zerklüftet und unübersichtlich. Öffnungsklauseln der verschiedensten Art sind inzwischen so verbreitet, dass schon die Steuerungskraft des ganzen Systems angezweifelt wird - vor allem in Gewerkschaftskreisen, aber auch in der Wissenschaft. Diese Kritik beruht aber auf einer zu engen und traditionellen Vorstellung von Steuerung. Analysiert man das breite Spektrum der im Laufe der Zeit entwickelten Kooperationsmodelle, z. B in der chemischen Industrie oder zuletzt in der Metall- und Elektroindustrie die „Pforzheimer Vereinbarung", so zeigen sich ganz neue Steuerungsmöglichkeiten, die mit den klassischen Instrumenten unerreichbar gewesen wären. Nicht nur Arbeitsbedingungen als Kostenfaktoren, sondern auch Organisationsstrukturen werden zum Gegenstand 
kollektiver Kooperation. Das ist nur auf der betrieblichen Ebene möglich.

Die Gewerkschaften bemühen sich verstärkt und auch mit Erfolg, die sozialen Folgen von Betriebsänderungen und -verlagerungen abzumildern, z. B. durch tarifliche Übergangsfristen, Abfindungen und Qualifizierungspflichten. Lediglich aus Kostengründen geplante Betriebsverlagerungen in das Ausland sollen durch Erhöhung der Verlagerungskosten erschwert oder gar verhindert werden. Das hat zu einer lebhaften Diskussion im Schrifttum geführt. (Für einen Überblick zur Diskussion Dieterich 2008, Art. 9, Rn. 75f.). Da wird argumentiert, Tarifverträge dieser Art seien nach Inhalt und Wirkung Sozialplänen vergleichbar. Dafür sei einzig das im Betriebsverfassungsgesetz geregelte Verfahren mit begrenzten Sanktionen vorgesehen, das allein der Unternehmerfreiheit gerecht werde. Die ausschließlich zuständigen Betriebsräte dürften nicht von den Tarifvertragsparteien verdrängt werden. Diese Diskussion ist eine symptomatische Erscheinungsform vielfältiger Bemühungen von politischer Seite, unterstützt von Stimmen aus der Wirtschafts- und der Rechtswissenschaft, das Verhältnis der beiden kollektiven Regelungssysteme zu verschieben. Ziel ist es, den Primat der Tarifautonomie zu relativieren und Tarifverträge auf betriebsferne Themenfelder oder Abstraktionshöhen abzudrängen. Aber dafür lässt sich die aktuelle Gesetzeslage nicht in Anspruch nehmen, wie das BAG mit einem jüngst gefällten Urteil zu Tarifsozialplänen festgestellt hat (BAG 2006).

\section{TARIFWIDRIGE BÜNDNISSE FÜR ARBEIT}

Das tarifpolitische Ideal ,kontrollierter Dezentralisierung" stößt jedoch auf große Widerstände in der Betriebspraxis. Jede Kooperation verlangt bekanntlich Zeit und Konzessionsbereitschaft. Aber beides können oder wollen viele Unternehmen nicht aufbringen, wenn sie eine schnelle Kostenentlastung für erforderlich halten und wenn sie auch ihre eigenen Betriebsräte von der Notwendigkeit einer Tarifabweichung überzeugt haben. So kommen betriebliche Regelungen zustande, die werbewirksam „Bündnisse für Arbeit“ genannt worden sind, deren Besonderheit aber lediglich darin besteht, dass sie ohne Mitwirkung der Tarifvertragsparteien maßgebende Tarifstandards unterschreiten. Manchmal wird wenigstens nachträglich deren Genehmigung erbeten, und auch das hat die Rechtsprechung als noch ausreichend akzeptiert (BAG 1999). Aber viele tarifgebundene Betriebe (Schätzungen rechnen mit ca. $15 \%$ ) machen sich nicht einmal diese Mühe und riskieren den offenen Tarifbruch. Das ist zwar rechtswidrig und in einem Rechtsstaat mit funktionierender Justiz auch ziemlich riskant, gilt aber neuerdings als innovativ. Ermutigt wird diese Praxis von Ökonomen, die die Marktrationalität kollektivvertraglicher Bindungen grundsätzlich in Zweifel ziehen.

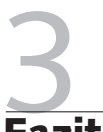

\section{Fazit}

Wo Ratlosigkeit herrscht, sind Patentrezepte wohlfeil und auch immer gleich auf dem Markt der Meinungen. Immer noch vorherrschend in der öffentlichen Debatte in Deutschland, wenn auch in etwas abnehmendem Maße, sind radikal markt-liberale Positionen. Ihre Vertreter werfen dem Sozialstaat vor, zu teuer und bürokratisch uneffizient zu sein. Gesetzliche Eingriffe, die die unternehmerische Gestaltungsfreiheit schmälern und sozialpolitisch motivierte Kosten verursachen, halten sie generell für kontraproduktiv; verbindliche Kollektivverträge bewerten sie als marktfeindliche Kartelle. So gesehen erscheinen die aktuellen Funktionsstörungen des Arbeitsrechts nur als Symptome einer anhaltenden Fehlsteuerung, oder genauer: unsinniger Steuerungsversuche. Der Staat sollte nach ihrer Ansicht seine sozial- oder arbeitsmarktpolitischen Bemühungen besser einstellen und das schadhaft gewordene Regelungsgebäude abreißen.

Dieser Ansatz verfehlt die sozialstaatlichen Aufgaben des Rechts. Wettbewerb ist zwar ein hervorragender Generator für Kreativität und Vitalität, und die Mechanismen eines freien Marktes sind für eine funktionierende Wirtschaft ganz unverzichtbar. Aber das ist nicht alles. Zum einen sind bedeutende Bereiche des gesellschaft- lichen Lebens ökonomischen Erwägungen entzogen, z. B. Wissenschaft und Kunst. Zum anderen führen die Marktgesetze als einziger Steuerungsmechanismus zwangsläufig zu Ergebnissen, die den Gerechtigkeitsvorstellungen der Gesellschaft widersprechen. Sie sind nämlich blind für moralische Kategorien. Die Dynamik der Vermögensbildung und -verteilung ist für sie kein Problem. Die Benachteiligung von Frauen und Minderheiten erscheint ihnen „marktgerecht“. Krankheit, Alter und Tod interessieren sie nur als Störfaktoren. Ohne die Beachtung sozialethischer Mindeststandards - Menschenwürde, soziale Gerechtigkeit, Sicherheit - gibt es keinen gesellschaftlichen Frieden. Dieser ist aber unverzichtbare Voraussetzung einer planungsfähigen Wirtschaft, intakter Infrastruktur und eines günstigen Investitionsklimas. Seine rechtliche Absicherung ist also keineswegs Luxus. Die Wertvorstellungen einer Gesellschaft sind auch nicht aus reinen Zweckmäßigkeitserwägungen manipulierbar. Wer demgegenüber auf den „homo öconomicus“ vertraut, einem Wesen, das sich jederzeit Marktgesetzen flexibel anpassen könnte und bereit wäre, alle persönlichen Konsequenzen kritiklos zu akzeptieren, huldigt einem Aberglauben. Der „homo öconomicus“ ist der Yeti der Wirtschaftheorie, im wirklichen Leben ist er nicht zu finden - nirgends.

Die Erfahrung lehrt zudem, dass wirtschaftliche Macht genauso die individuelle Freiheit bedroht, also der Begrenzung bedarf, wie staatliche Macht. Das ist der Grund, warum sich die Bundesrepublik als Sozialstaat bezeichnet und damit die Gerechtigkeitserwartungen unserer Gesellschaft auf den Punkt bringt. Das Recht muss dies umsetzen, indem es widerstreitende Interessen ausgleicht und dem Machtmissbrauch Grenzen setzt - nicht mehr, aber auch nicht weniger.

Richtig ist allerdings, dass gesellschaftliche Wertungen sich ändern und auch veränderten Realitäten anpassen. Deshalb ist die Frage nach der rechtlichen Rahmenordnung in einer Zeit supranationaler Strukturen neu zu stellen. Eine Antwort darauf versucht Pfarr im nachfolgenden Artikel. 


\section{LITERATUR}

Dieterich, T. (2008): Grundgesetz, in: Dieterich, T./Müller-Glöge, R./ Preis, U./Schaub, G. (Hrsg.): Erfurter Kommentar zum Arbeitsrecht, 8. Aufl., München (im Erscheinen)

\section{Rechtsprechung}

Bundesarbeitsgericht (BAG) (1987): Beschluss vom 18.8.1987, in: Der Betrieb 44, S. 2257-2262
Bundesarbeitsgericht (BAG) (1999): Urteil vom 20.4.1999, in: Der Betrieb 32, S. 1660-1663

Bundesarbeitsgericht (BAG) (2006): Urteil vom 6.12.2006, in: Der Betrieb 24, 2007, S. 1362-1364; zur Erstreitbarkeit BAG 24.4.2007 - 1 AZR 252/06 